\title{
Impairment of Social Function in Young Females With Recent-Onset Anorexia Nervosa and Recovered Individuals
}

\author{
Mette Bentz, M.S. ${ }^{\text {a,b,* }}$, Jens Richardt Moellegaard Jepsen, Ph.D. ${ }^{\text {a,c,d }}$, Tine Pedersen, M.S. ${ }^{\text {a,b,e }}$, \\ Cynthia M. Bulik, Ph.D. ${ }^{\mathrm{f}, \mathrm{g}, \mathrm{h}}$, Lennart Pedersen, M.S. ${ }^{1}$, Anne Katrine Pagsberg, M.D., Ph.D. ${ }^{\mathrm{a}, \mathrm{b}}$, and \\ Kerstin J. Plessen, M.D., Ph.D. ${ }^{\text {a,b }}$ \\ ${ }^{a}$ Child and Adolescent Mental Health Centre, Mental Health Services in the Capital Region of Denmark, Copenhagen, Denmark \\ ${ }^{\mathrm{b}}$ Department of Clinical Medicine, Faculty of Health and Medical Sciences, University of Copenhagen, Copenhagen, Denmark \\ ${ }^{c}$ Lundbeck Foundation Center for Clinical Intervention and Neuropsychiatric Schizophrenia Research (CINS), Psychiatric Center, Glostrup, Denmark \\ ${ }^{\text {d } C e n t e r ~ f o r ~ N e u r o p s y c h i a t r i c ~ S c h i z o p h r e n i a ~ R e s e a r c h ~(C N S R), ~ P s y c h i a t r i c ~ C e n t e r, ~ G l o s t r u p, ~ D e n m a r k ~}$ \\ e Danish Research Centre for Magnetic Resonance, Centre for Functional and Diagnostic Imaging and Research, Copenhagen University Hospital Hvidovre, Hvidovre, Denmark \\ ${ }^{\mathrm{f}}$ Department of Psychiatry, University of North Carolina at Chapel Hill, Chapel Hill, North Carolina \\ ${ }^{\mathrm{g}}$ Department of Nutrition, University of North Carolina at Chapel Hill, Chapel Hill, North Carolina \\ ${ }^{\mathrm{h}}$ Department of Medical Epidemiology and Biostatistics, Karolinska Institutet, Stockholm, Sweden \\ ${ }^{\mathrm{i}}$ Center for Autisme, Herlev, Denmark
}

Article history: Received January 24, 2016; Accepted August 4, 2016

Keywords: Anorexia nervosa; Social function; Social cognition; Autistic traits; Recovered; Adolescents

\section{A B S T R A C T}

Purpose: A subgroup of individuals with anorexia nervosa (AN) displays social difficulties; however, it is not clear if individuals with comorbid autism spectrum disorders account for these difficulties.

Methods: We compared social function using the Autism Diagnostic Observation Schedule in 43 young females with first-episode AN who did not have comorbid autism spectrum disorder, 28 individuals recovered from adolescent-onset AN, and 41 healthy comparison individuals (age range 14-22 years). We measured adaptive behavior with the Vineland-II parent questionnaire, and aspects of social cognition with psychological tests, such as the Reading-the-Mind-in-the-Eyes test, Profile of Nonverbal Sensitivity short version, The Awareness of Social Inference Test, Animated Triangles, and the CANTAB Affective Go/No-go task.

Results: Participants with first-episode AN and those recovered from AN displayed difficulties in social function, which were not associated with body mass index or other state factors of the disorder in those with first-episode AN. Mood problems and anxiety were not associated with these difficulties. Parents rated participants with first-episode AN lower than recovered and control participants on the Socialization Domain of Vineland-II. Finally, only participants recovered from AN demonstrated deficits in specific domains of social cognition: perceiving nonverbal bodily gesture and vocal prosody.

Conclusions: Young females with first-episode AN and those recovered from AN displayed impairments in social function, which may represent more stable traits of the disorder. Only participants recovered from AN demonstrated deficits in social cognition.

\section{IMPLICATIONS AND \\ CONTRIBUTION}

On the group level, young females with anorexia nervosa without autism spectrum disorders displayed impaired social function but no inferior social cognition capacity. Recovered individuals were impaired in their social function and showed few social cognitive deficits. State factors of the illness did not explain impairment of social function.

\footnotetext{
Conflicts of Interest: The authors report no conflicts of interest.

* Address correspondence to: Mette Bentz, M.S., Child and Adolescent Mental Health Centre, Mental Health Services in the Capital Region of Denmark, Bispebjerg Bakke 30, 2400 København NV, Denmark.

E-mail address: mette.bentz@regionh.dk (M. Bentz).
} 
Social difficulties are inherent in current models of vulnerability and maintenance of anorexia nervosa (AN) [1], and a better understanding of these may refine interventions and improve outcome for this serious disorder. However, evidence of social difficulties in young individuals with a recent onset AN and in those recovered from AN is limited. We studied these difficulties at two descriptive levels: social function and social cognition.

Social function encompasses interactive behavior and capacity for socioemotional reciprocity [2]. Social cognition is broadly defined as the mental operations underlying social interaction [3]. Deficits in social cognition are documented in individuals with AN $[4,5]$. However, the relationship between behavioral and cognitive aspects of social difficulties is not clear, and instruments to reliably quantify difficulties in social function are scarce. The Autism Diagnostic Observation Schedule (ADOS) measures observable interactive behavior in a standardized form [6]. It is intended to qualify a diagnosis of autism spectrum disorder (ASD), a spectrum of developmental disorders characterized by sociocommunicative deficits, and mental and behavioral inflexibility. However, the ADOS quantifies behaviors without assumptions of possible underlying mechanisms, and we therefore used the ADOS to assess social function in AN.

We investigated social function in young females with firstepisode AN and those recovered from adolescent-onset AN, both groups without comorbid ASD. In exploratory analyses, we assessed social function via parent reports and social cognition via experimental tests. Finally, we explored associations between social function and social cognition across diagnostic groups.

We hypothesized that young females with first-episode AN would show impairments in social function and that those recovered from AN would perform intermediate between those with first-episode AN and controls.

\section{Methods}

\section{Subjects}

We invited young females with a recent onset of their first episode of AN (International Classification of Diseases [ICD-10]: F50.0 or F50.1) from the Centre for Child and Adolescent Mental Health Services (CAMHS; 14 - to 17-year-old patients), the Capital Region of Denmark. Recent onset was defined as onset within a maximum of 12 months. Prior psychological treatment was allowed but infrequent, as CAMHS is usually the first line of treatment in Denmark. Inclusion criteria for this group included a body mass index (BMI) percentile corrected for age $<25$ th for $14-15$ year olds and a BMI $<18.5 \mathrm{~kg} / \mathrm{m}^{2}$ for participants 16 years or older. All eligible CAMHS patients between July 2012 and March 2015 were invited if possible. In addition, we recruited a few young adults with first-episode AN from the Stolpegaard Psychotherapy Centre (18- to 21-year-old patients), the Capital Region of Denmark. Our first-episode AN participants thus were representative of a young population with a recent onset, without substantial epiphenomena of chronicity.

Second, we invited young females recovered from AN (ICD-10: F50.0 or F50.1) with onset in late childhood or adolescence. They were identified through a quality assurance survey. Recovery was defined as Eating Disorders Examination (EDE) global score within one standard deviation (SD) of non-AN norms [7], $\geq 9$ points on the Morgan Russell Outcome Assessment Schedule [8], and no current eating disorder pathology or current treatment for an eating disorder. We established the absence of eating disorder pathology for the last 3 months with EDE diagnostic questions and normal body weight for the last 12 months via self-report.

Finally, we invited control participants via advertisements in state schools, halls of residence, and colleges in the catchment area of the hospital. Inclusion criteria were a normal body weight and absence of psychiatric disorders throughout their lifetime (minor exceptions included transient childhood tics and adjustment disorders).

Comorbidity is well documented during and after the course of AN [9], and accordingly, we did not exclude recovered individuals with past or present psychopharmacological treatment. To address our primary question of whether social deficits were confined to individuals with comorbid ASD, exclusion criteria further included ASD for all three groups. As a screening for undetected ASD, parents completed the Social Communication Questionnaire [10] and the Asperger Syndrome Screening Questionnaire-Revised Extended Version [11]. If ratings were above the established cutoffs, we interviewed parents with the Autism Diagnostic Interview-Revised [12]. Combined, these procedures ensured that no participants fulfilled diagnostic criteria for childhood autism (F84.0) or Asperger syndrome (F84.5).

We assessed psychiatric symptoms with the Schedule for Affective Disorders and Schizophrenia for School-Age Children, Present and Lifetime version [13] and the Beck Youth Inventory [14], and intelligence with the Reynolds Intellectual Assessment Scales [15]. Clinical data at the time of treatment for AN were available for the recovered participants, enabling comparison of AN severity at onset between the clinical groups. Finally, the regional Scientific Ethical Committees (project number $\mathrm{H}-2$ 2012-027) and the Danish Data Protection Agency approved the study, and participants and legal caretakers gave informed consent according to the guidelines of the Danish Health and Medicines Authority.

\section{Outcome measures}

We measured social function with the ADOS-2, Danish version, module 4 [6]. During the ADOS observation, the observer orchestrates a series of situations with a social press for communication and interaction. The ADOS algorithm yields two subscales: Communication and Reciprocal Social Interaction, and a "Communication and Social Interaction Total" (ADOS-Total). Our main outcomes were the ADOS-Total and the two subscales. In module 4 , the clinical cutoff for ADOS-Total is $\geq 7$, for the Communication Subscale is $\geq 2$, and for the Reciprocal Social Interaction Subscale is $\geq 4$. These cutoffs represents a $90 \%$ sensitivity and a 93\% specificity for distinguishing ASD from nonspectrum cases [16].

Interrater reliability of the main rater and an experienced ADOS-certified psychologist blind to group was monitored in 15 randomly chosen cases, and weighted Kappa for all items was satisfactory (.77).

We used the Socialization Domain of Vineland-II [17] as an additional probe of parent-reported social function measuring adaptive behaviors in everyday life.

We documented social cognitive performance with tests tapping into the following four subdomains identified by the Measurement and Treatment Research to Improve Cognition in Schizophrenia [18]. 
Emotion perception. "Reading the Mind in the Eyes-Revised" (RME-R) [19] displays photos of the eye region and words describing complex feelings.

Social perception. The "MiniPONS" [20], a short version of the Profile of Nonverbal Sensitivity test, consists of 64 short film clips of body gestures, facial expressions, and/or vocal prosody.

Theory of Mind (ToM). Twelve cartoons of Animated Triangles (AT) [21] were presented in a fixed, random order using the multiple-choice response format [21]. The Awareness of Social Inference Test (TASIT), Part 2: Social interference-Minimal, Danish version [22], presents short movies with either sincere, simple sarcastic, or paradoxical sarcastic interactions.

Affective bias. CANTAB Affective Go/No-go (AGN) [23] consists of briefly displayed positive and negative words where target category alternates.

We translated phrases and questions for the RME-R, MiniPONS, and AT into Danish in agreement with the authors of the tests. Tests were presented in a fixed order along with interviews and questionnaires during 2-3 appointments, scheduled as soon as possible.

\section{Statistical procedures}

Statistical analyses were carried out in IBM SPSS Statistics version 22 (IBM Corp., Armonk, New York). We tested our main hypothesis with ADOS-Total, Communication Subscale, and Reciprocal Social Interaction Subscale as dependent variables and group as independent variable using analyses of variance (ANOVAs). If assumptions for parametric analyses were not met, the test was replaced by nonparametric alternatives, that is, independent samples Kruskal-Wallis tests. We used Bonferroni correction of the alpha level according to three main analyses ( $p$ $<.017$ ) to control the type I error, and we used independent samples Mann-Whitney $U$ Tests for post hoc analyses. ADOS scores were not adjusted for age because of age independence of the algorithms of ADOS, module 4 [24].

All remaining analyses were regarded as exploratory. We used Spearman's $\rho$ to analyze the association between ADOS-Total and Beck Depression Inventory Youth and Beck Anxiety Inventory Youth within each group. We explored the association of social function with body weight and symptom severity among individuals with first-episode AN by calculating bivariate correlations of the ADOS-Total with BMI adjusted for age, EDE global score, and each of the four EDE subscales.

We used independent samples Kruskal-Wallis tests and a forest-type plot to detail profiles of single-item differences in ADOS. Vineland-II Socialization domain Index scores was analyzed with one-way ANOVA. We weighted cases with a standardized frequency weight based on four age groups, to adjust the social cognitive test scores for group difference in age. Preliminary inspections revealed substantial ceiling effects, and consequently we excluded items, which 95\% of controls answered correctly ( 2 of 36 RME-R photos; 17 of 64 MiniPONS clips; 5 of 15 TASIT films; 8 of 12 AT film-type questions). We used ANOVAs to investigate between-group differences of these reduced test sets and followed up with Tukey or Games-Howell post hoc test. We assessed normality of residuals by inspection of Q-Q plots and inspected box plots to secure there were no extreme outliers.
Affective bias was calculated as difference between mean response time of positive and negative blocks of the AGN. Finally, to explore associations between social function and social cognition, we entered those social cognition test variables showing group difference and age as independent variables in an ordinal logistic regression (proportional odds) with ADOS-Total cut into categories as the dependent variable, as model assumptions for ADOS-Total as continuous outcome was questionable. The categories were zero (no observed deviation), low (scores of 1 through 3 ), medium (scores of 4 through 6 ), and high (scores above clinical cutoff, i.e., $\geq 7$ ). Interaction terms with groups were inspected, and if significant, groups were to be analyzed separately.

\section{Results}

\section{Subjects}

In total, 43 first-episode AN participants, 28 recovered participants, and 41 healthy participants agreed to participate (Table 1). Mean weight gain among individuals with acute AN from clinical assessment to first testing session was $2.1 \mathrm{~kg}$ (median $=1.7 \mathrm{~kg}, \mathrm{SD}=2.3$ ), and mean duration from first to last visit was 8.0 days (median $=7.0$ days, range $=1-23$ days, $\mathrm{SD}=6.3$ ). Duration from first to last visit was similar between groups (recovered participants mean $=5.6$ days [median $=$ 3 days, range $=1-28, \mathrm{SD}=6.7$ ], controls mean $=8.2$ days [median $=6.5$, range $=1-35,=$ SD 8.2], independent samples Kruskal-Wallis Test: $\left.\chi^{2}[2]=3.866, p=.15\right)$. Current comorbidity was comparable across first-episode AN participants and those recovered from AN (Table 1 ). The three groups did not differ with respect to intelligence, parental education, or family structure; however, the group of recovered participants was older (Table 1).

Severity of illness at onset was comparable between the diagnostic groups; they had similar mean BMI percentiles corrected for age at onset (first-episode $\mathrm{AN}=3.84[\mathrm{SD}=5.4]$; recovered $=4.83[\mathrm{SD}=6.0]$ ), rate of AN subtypes (binge/purge: first-episode $\mathrm{AN}=9 \%$; recovered $=14 \%$ ), rate of comorbid depression (first-episode $\mathrm{AN}=18.6 \%$; recovered $=14.3 \%$ ), and mean EDE scores (Table 1) when first presenting to the clinic. However, the recovered participants had a lower mean age at time of treatment onset (Table 1).

\section{Primary analyses of social function}

Participants with first-episode AN and those recovered from AN demonstrated higher mean rank of ADOS-Total scores than controls (independent samples Kruskal-Wallis Test: $\chi^{2}$ $[2]=8.277, p=.002$; Table 2 and Figure 1 ). Moreover, groups differed in mean rank of their Reciprocal Social Interaction Subscale score (independent samples Kruskal-Wallis Test: $\chi^{2}$ $[2]=11.954, p=.003$; Table 2). The two clinical groups did not differ on any ADOS scale. A subgroup of participants with firstepisode $A N(N=7 ; 16 \%)$ and of those recovered $(N=6 ; 21 \%)$ exceeded an ADOS-Total score above the clinical cutoff for an autism spectrum classification according to the ADOS system $(\geq 7)$, and most of these (first-episode $A N$ : $N=6$, recovered: $\mathrm{N}=4$ ) also exceeded the clinical cutoff on each subscale separately (Communication [ $\geq 2]$, Social Interaction $[\geq 4]$ ), suggesting two distinguishable subgroups: "normal" scorers and "high" scorers on the ADOS-Total. None of the control participants exceeded clinical cutoff on the ADOS classification (Table 2). 


\begin{tabular}{|c|c|c|c|c|c|}
\hline & $\begin{array}{l}\text { First-episode } \\
\text { AN }(N=43)\end{array}$ & $\begin{array}{l}\text { Recovered } \\
\text { AN }(N=28)\end{array}$ & $\begin{array}{l}\text { Controls } \\
(\mathrm{N}=41)\end{array}$ & Test statistics & Pairwise comparison $(p)$ \\
\hline Age in years, mean (SD) & $16.1(1.5)$ & $18.4(1.6)$ & $17.7(2.2)$ & $\begin{array}{l}\text { ANOVA F }(2,109): 16.119 \\
p<.001\end{array}$ & $\begin{array}{l}\text { First-episode }<\text { recovered } p<.001 \\
\text { First-episode }<\text { controls } p<.001 \\
\text { Recovered-controls ns }\end{array}$ \\
\hline $\begin{array}{l}\text { Age at time of treatment, } \\
\text { mean }(\mathrm{SD})\end{array}$ & $16.1(1.5)$ & $14.8(1.6)$ & $\mathrm{n} / \mathrm{a}$ & $\begin{array}{l}\text { Independent Samples } \mathrm{t}(69): 3.357 \\
p=.001\end{array}$ & $\begin{array}{l}\text { First-episode }>\text { recovered } p=.001 \\
\text { First-episode-controls ns } \\
\text { Recovered-controls ns }\end{array}$ \\
\hline $\begin{array}{l}\text { Parents' highest education, } \\
\text { years, mean (SD) }\end{array}$ & $16.1(2.3)$ & $14.8(2.8)$ & $15.3(2.4)$ & $\begin{array}{l}\text { ANOVA F }(2,109): 2.591 \\
p=.08\end{array}$ & $\begin{array}{l}\text { First-episode-recovered ns } \\
\text { First-episode-controls ns } \\
\text { Recovered-controls ns }\end{array}$ \\
\hline $\begin{array}{l}\text { Living with both parents } \\
\text { together, } \mathrm{N}(\%)\end{array}$ & $24(56 \%)$ & $17(61 \%)$ & $21(51 \%)$ & $\begin{array}{l}\text { Kruskal-Wallis: } \chi^{2}(2)=.613 \\
p=.74\end{array}$ & $\begin{array}{l}\text { First-episode-recovered ns } \\
\text { First-episode-controls ns } \\
\text { Recovered-controls ns }\end{array}$ \\
\hline BMI $\left(\mathrm{kg} / \mathrm{m}^{2}\right)$, mean (SD) & $16.6(1.2)$ & $21.3(1.8)$ & $22.0(2.6)$ & $\begin{array}{l}\text { ANOVA F }(2,109): 92.608 \\
p<.001\end{array}$ & $\begin{array}{l}\text { First-episode }<\text { recovered } p<.001 \\
\text { First-episode }<\text { controls } p<.001 \\
\text { Recovered-controls ns }\end{array}$ \\
\hline $\begin{array}{l}\text { BMI percentile corrected for } \\
\text { age, mean }(\mathrm{SD})^{\mathrm{a}}\end{array}$ & $7.5(7.6)$ & $47.3(19.0)$ & $56.9(21.2)$ & $\begin{array}{l}\text { ANOVA F }(2,109): 101.948 \\
p<.001\end{array}$ & $\begin{array}{l}\text { First-episode }<\text { recovered } p<.001 \\
\text { First-episode }<\text { controls } p<.001 \\
\text { Recovered-controls ns }\end{array}$ \\
\hline $\begin{array}{l}\text { EDI eating disorder risk composite, } \\
\text { mean }(\mathrm{SD})^{\mathrm{b}}\end{array}$ & $47.7(10.1)$ & $36.5(6.4)$ & $36.1(7.0)$ & $\begin{array}{l}\text { ANOVA F }(2,105): 24.861 \\
p<.001\end{array}$ & $\begin{array}{l}\text { First-episode }>\text { recovered } p<.001 \\
\text { First-episode }>\text { controls } p<.001 \\
\text { Recovered-controls ns }\end{array}$ \\
\hline $\begin{array}{l}\text { EDE global score at time of treatment, } \\
\text { mean }(S D)^{c}\end{array}$ & $2.8(1.5)$ & $2.8(1.3)$ & $\mathrm{n} / \mathrm{a}$ & $\begin{array}{l}\text { Independent Samples t-test } \\
\mathrm{t}(54):-.051 p=1.0\end{array}$ & First-episode-recovered ns \\
\hline $\begin{array}{l}\text { EDE global score at time of testing, } \\
\text { recovered participants, mean (SD) }\end{array}$ & $\mathrm{n} / \mathrm{a}$ & $.6(.5)$ & $\mathrm{n} / \mathrm{a}$ & & \\
\hline $\begin{array}{l}\text { Duration of treatment for recovered } \\
\text { participants (months) mean (SD) }\end{array}$ & $\mathrm{n} / \mathrm{a}$ & $23.9(11.8)$ & $\mathrm{n} / \mathrm{a}$ & & \\
\hline $\begin{array}{l}\text { General intelligence quotient, RIAS, } \\
\text { mean (SD) }\end{array}$ & $107.7(10.5)$ & $102.8(11.5)$ & $107.3(9.5)$ & $\begin{array}{l}\text { ANOVA F }(2,109): 2.137 \\
p=.12\end{array}$ & $\begin{array}{l}\text { First-episode-recovered ns } \\
\text { First-episode-controls ns } \\
\text { Recovered-controls ns }\end{array}$ \\
\hline Comorbid depressive disorder, $\mathrm{N}(\%)^{\mathrm{d}, \mathrm{e}}$ & $8(19)$ & $3(11)$ & & Fishers exact (2-sided) & .51 \\
\hline Comorbid OCD, N (\%) & $3(7)$ & $0(0)$ & & Fishers exact (2-sided) & .27 \\
\hline Comorbid anxiety other than OCD, N (\%) & $4(9)$ & $5(18)$ & & Fishers exact (2-sided) & .30 \\
\hline
\end{tabular}

$\mathrm{BMI}=$ body mass index; EDE = Eating Disorder Examination; EDI = Eating Disorder Inventory-3; n/a = not applicable; ns = non-significant; RIAS = Reynolds Intellectual Assessment Scales.

${ }^{\mathrm{a}}$ BMI percentiles corrected for age $<.02$, calculated as $=.02$. Participants $>20 \mathrm{yr}$ old given percentile of $20 \mathrm{yr}$.

b EDI-3 answers are missing from four FeAN.

c EDE data available from time of treatment for recovered participants, $\mathrm{N}=13$ (46\%). T-scores are derived from American norms in the absence of Danish norms for adolescents.

e Depressive disorder includes mild depression, moderate depression and severe depression according to ICD-10.

f We used the term "comorbid" although anxiety and depression are only comorbid to AN in the case of the participants with first-episode AN.

\section{Exploratory analyses}

In bivariate within-group analyses, ADOS-Total showed nonsignificant correlations with self-reported symptoms of depression and of anxiety. However, there was a trend toward a significant correlation between ADOS-Total scores and symptoms of depression in the first-episode AN group (Table 3). In bivariate analyses within first-episode AN participants, ADOS-Total scores were not associated with BMI percentile, EDE global score, nor EDE subscales (Table 3).

Participants with first-episode AN differed from controls concerning the algorithm items "Offers Information," "Emphatic or Emotional Gestures," "Facial Expressions Directed to Others," "Communication of Own Affect," "Amount of Social Overtures," "Amount of Reciprocal Social Communication" and "Overall Quality of Rapport." Recovered participants differed from controls concerning the items "Offers Information," "Reporting of Events," "Facial Expressions Directed to Others," "Communication of Own Affect," "Amount of Social Overtures," "Amount of Reciprocal Social Communication," and “Overall Quality of Rapport” (Table 3; Figure 2).

\section{Vineland-II}

Participants with first-episode AN, but not those recovered, were reported to have reduced adaptive function with a small effect size (Table 3). The correlation between ADOS-Total and Vineland-II Socialization Domain was small $(r=-.2$, $p=.03)$.

\section{Social cognition}

Groups differed in specific aspects of social perception: MiniPONS body gestures with a medium effect size $\left(R^{2}=.14\right)$, and MiniPONS vocal prosody with a small effect size $\left(R^{2}=.07\right.$; Table 3). Post hoc tests showed that recovered participants performed worse than first-episode AN participants in these two tests (Cohen's $d=-0,89$ and -.64 , respectively) and had lower scores than controls in MiniPONS body gestures (Cohen's $d=-.77$ ). However, groups did not differ regarding the two other classes of stimuli in the MiniPONS (facial expression, and facial expression and vocal prosody), nor regarding RME-R, TASIT, AT, or AGN. Participants with first-episode AN performed similar to control participants on all tests of social cognition.

\section{Association between social function and social cognition}

In an ordinal regression model for prediction of ADOS-Total category, neither age, MiniPONS body gestures, nor MiniPONS 
Table 2

Main analyses of group differences in ADOS

\begin{tabular}{|c|c|c|c|c|c|c|}
\hline ADOS algorithm scores & $\begin{array}{l}\text { First-episode } \\
\text { AN }(N=43)\end{array}$ & $\begin{array}{l}\text { Recovered } \\
\text { AN }(N=28)\end{array}$ & $\begin{array}{l}\text { Controls } \\
(\mathrm{N}=41)\end{array}$ & Test statistics $^{\mathrm{a}}$ & \multicolumn{2}{|c|}{$\begin{array}{l}\text { Post hoc comparisons }{ }^{b} \text { (unadjusted } \\
p \text { value, effect size }{ }^{c} \text { ) }\end{array}$} \\
\hline $\begin{array}{l}\text { ADOS-Total (= ADOS Communication and } \\
\text { Social Interaction); mean (SD), mean rank }\end{array}$ & $2.77(3.1), 63.5$ & 2.79 (3.2), 62.1 & 1.05 (1.5), 45.3 & $\chi^{2}(2)=8.277$ & .02 & $\begin{array}{l}\text { First episode-recovered: ns } \\
\text { First episode }>\text { controls: } .008 \text {, } \\
\quad \text { abs }(r)=.29 \\
\text { Recovered }>\text { controls: } .03 \text {, } \\
\quad \text { abs }(r)=.27\end{array}$ \\
\hline $\begin{array}{l}\text { ADOS Communication Subscale; mean (SD), } \\
\text { mean rank }\end{array}$ & 1.35 (1.6), 61.0 & 1.36 (1.6), 61.8 & $.66(1.0), 48.1$ & $\chi^{2}(2)=4.928$ & .09 & \\
\hline $\begin{array}{l}\text { ADOS Reciprocal Social Interaction Subscale; } \\
\text { mean (SD), mean rank }\end{array}$ & $1.42(1.8), 64.7$ & $1.43(1.8), 62.3$ & $.39(.9), 44.0$ & $\chi^{2}(2)=11.954$ & .003 & $\begin{array}{l}\text { First episode-recovered: ns } \\
\text { First episode }>\text { controls: } .001 \text {, } \\
\quad \text { abs }(r)=.37 \\
\text { Recovered }>\text { controls: } .01 \text {, } \\
\quad \text { abs }(r)=.31\end{array}$ \\
\hline $\begin{array}{l}\text { Participants with ADOS-Total exceeding } \\
\text { cutoff }(\geq 7), N(\%)\end{array}$ & $7(16 \%)$ & $6(21 \%)$ & $0(0 \%)$ & & & \\
\hline
\end{tabular}

ADOS = Autism Diagnostic Observation Schedule; $\mathrm{AN}=$ anorexia nervosa; $\mathrm{ns}=$ nonsignificant; $\mathrm{SD}=$ standard deviation

a Independent samples Kruskal-Wallis test unless otherwise specified.

b Mann-Whitney $U$ test.

${ }^{c}$ Effect size calculated as $r=$ standardized test statistic $/ \sqrt{ } \mathrm{N}$, reported in absolute values.

vocal prosody was significant predictors, and only group was a significant factor.

\section{Discussion}

Participants with first-episode AN and recovered from AN displayed similar degrees of impairments of social function on the group level, confirming that social deficits in AN are not limited to the acute state of illness-a finding that was driven by a subgroup of participants in each clinical group. Moreover, participants recovered from AN demonstrated impairments of their perception of social stimuli, whereas participants with first-episode AN demonstrated normal performance on these measures.
The impairment in social function measured with ADOS highlights some similar traits between individuals with AN and those with ASD, although the social impairments we observed in most AN participants were smaller than those reported in ASD [6]. Elevated autistic traits have been confirmed in studies of adults with AN using other instruments, that is, questionnaires [25], or the ADOS [26]. Two studies assessed social function in young AN patients with other instruments and reported similar findings as ours [27,28]. However, our study extended and clarified these observations by using the ADOS instrument in a representative sample of young females with AN and by excluding individuals with known or detected comorbid ASD.
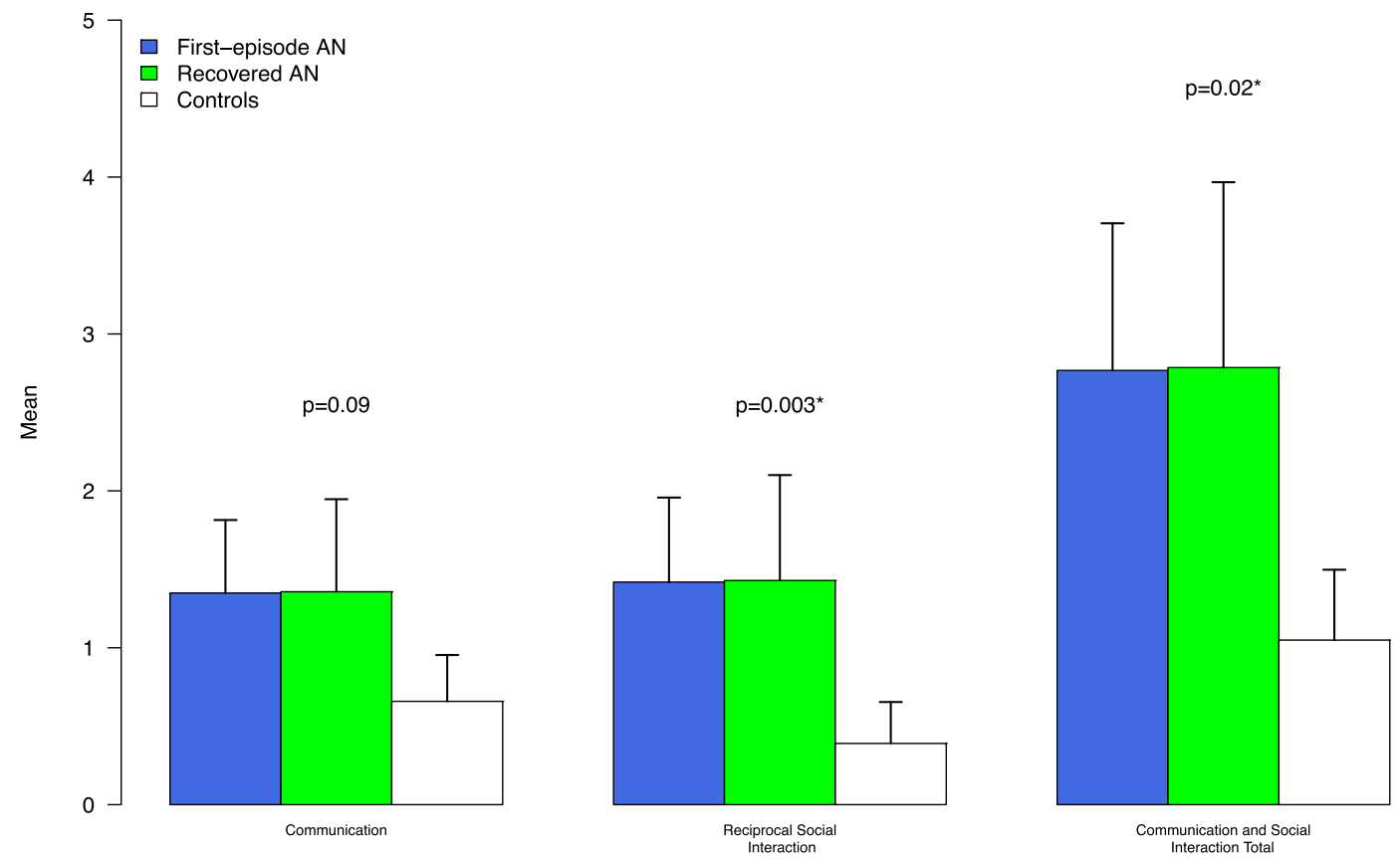

Figure 1. ADOS algorithm scores. " Significant group differences. Error bars represent $95 \%$ confidence interval. 


\begin{tabular}{|c|c|c|c|c|c|c|}
\hline Exploratory measures & $\begin{array}{l}\text { First-episode } \\
\text { AN }(N=43)\end{array}$ & $\begin{array}{l}\text { Recovered } \\
\text { AN }(\mathrm{N}=28)\end{array}$ & Controls $(\mathrm{N}=41)$ & Test statistics $^{\mathrm{a}}$ & $p$ & $\begin{array}{l}\text { Post hoc comparisons }{ }^{\mathrm{b}} \\
\text { (unadjusted } p \text {-value, effect size }{ }^{\mathrm{c}} \text { ) }\end{array}$ \\
\hline \multicolumn{7}{|c|}{ Supplementary ADOS subscales (beyond study hypotheses) } \\
\hline $\begin{array}{l}\text { ADOS Imagination/Creativity, } \\
\text { mean (SD) }\end{array}$ & $.51(.6)$ & $.68(.5)$ & $.32(.6)$ & Kruskal-Wallis $\chi^{2}(2): 7.114$ & .03 & $\begin{array}{l}\text { First-episode }<\text { recovered ns } \\
\text { First-episode }>\text { controls ns } \\
\text { Recovered }>\text { controls } p=.02\end{array}$ \\
\hline $\begin{array}{l}\text { ADOS Stereotyped behaviors and } \\
\text { restricted interests, } \mathrm{N} \text { with score }>0\end{array}$ & $\mathrm{~N}=1(2 \%)$ & $\mathrm{N}=0(0 \%)$ & $\mathrm{N}=0(0 \%)$ & $\chi^{2}(2): 1.619$ & .5 & \\
\hline \multicolumn{7}{|c|}{ Associations between social function and clinical variables (bivariate within-group correlations) } \\
\hline ADOS and BDI-Y & $r_{\mathrm{s}}=.29, p=.06$ & $r_{\mathrm{s}}=.10, p=.62$ & $r_{\mathrm{s}}=.11, p=.49$ & & & \\
\hline ADOS and BAI-Y & $r_{\mathrm{s}}=.25, p=.10$ & $r_{\mathrm{s}}=-.19, p=.35$ & $r_{\mathrm{s}}=-.003, p=.99$ & & & \\
\hline $\begin{array}{l}\text { ADOS and BMI percentile corrected } \\
\text { for age }\left(^{*}\right)\end{array}$ & $r_{\mathrm{s}}=-.03, p=.86$ & $\mathrm{n} / \mathrm{a}$ & $\mathrm{n} / \mathrm{a}$ & & & \\
\hline ADOS and EDE global score & $r_{\mathrm{s}}=.08, p=.61$ & $\mathrm{n} / \mathrm{a}$ & $\mathrm{n} / \mathrm{a}$ & & & \\
\hline ADOS and EDE subscale Restraint & $r_{\mathrm{s}}=.10, p=.54$ & $\mathrm{n} / \mathrm{a}$ & $\mathrm{n} / \mathrm{a}$ & & & \\
\hline $\begin{array}{l}\text { ADOS and EDE subscale } \\
\text { Eating Concern }\end{array}$ & $r_{\mathrm{s}}=.11, p=.51$ & $\mathrm{n} / \mathrm{a}$ & $\mathrm{n} / \mathrm{a}$ & & & \\
\hline $\begin{array}{l}\text { ADOS and EDE subscale } \\
\text { Weight Concern }\end{array}$ & $r_{\mathrm{s}}=.12, p=.53$ & $\mathrm{n} / \mathrm{a}$ & $\mathrm{n} / \mathrm{a}$ & & & \\
\hline ADOS and EDE subscale Shape Concern & $r_{\mathrm{s}}=.26, p=.14$ & $\mathrm{n} / \mathrm{a}$ & $\mathrm{n} / \mathrm{a}$ & & & \\
\hline \multicolumn{7}{|c|}{ ADOS single items: between-group differences ${ }^{\mathrm{d}}$} \\
\hline $\begin{array}{l}\text { ADOS item: A5, Offers Information, } \\
\text { mean rank }\end{array}$ & 58.65 & 64.09 & 49.06 & Kruskal-Wallis $\chi^{2}(2)=8.715$ & .01 & $\begin{array}{l}\text { First-episode AN }>\text { controls: } p=.03, \text { abs }(\mathrm{r})=.2 \\
\text { Recovered }>\text { controls: } p=.002, \text { abs }(\mathrm{r})=.4 \\
\text { First-episode AN }<\text { Recovered ns }\end{array}$ \\
\hline $\begin{array}{l}\text { ADOS item: A7, Reporting of Events, } \\
\text { mean rank }\end{array}$ & 54.88 & 71.11 & 48.22 & Kruskal-Wallis $\chi^{2}(2)=12.903$ & .002 & $\begin{array}{l}\text { First-episode AN }>\text { controls ns } \\
\text { Recovered }>\text { controls: } p<.001 \text {, abs }(\mathrm{r})=.4 \\
\text { First-episode } \mathrm{AN}<\text { Recovered: } p=.02 \text {, abs }(\mathrm{r})=.3\end{array}$ \\
\hline $\begin{array}{l}\text { ADOS item: A10, Emphatic or Emotional } \\
\text { Gestures, mean rank }\end{array}$ & 63.15 & 56.18 & 49.74 & Kruskal-Wallis $\chi^{2}(2)=6.090$ & .05 & $\begin{array}{l}\text { First-episode AN }>\text { controls: } p=.01 \text {, abs }(\mathrm{r})=.3 \\
\text { Recovered }>\text { controls ns } \\
\text { First-episode AN }>\text { Recovered ns }\end{array}$ \\
\hline $\begin{array}{l}\text { ADOS item: B2, Facial Expressions } \\
\text { Directed to Others, mean rank }\end{array}$ & 57.43 & 68.50 & 47.33 & Kruskal-Wallis $\chi^{2}(2)=11.641$ & .003 & $\begin{array}{l}\text { First-episode AN }>\text { controls: } p=.05, \text { abs }(\mathrm{r})=.2 \\
\text { Recovered }>\text { controls: } p=.001, \text { abs }(\mathrm{r})=.4 \\
\text { First-episode AN }<\text { Recovered ns }\end{array}$ \\
\hline $\begin{array}{l}\text { ADOS item: B5, Communication of } \\
\text { Own Affect, mean rank }\end{array}$ & 61.31 & 65.14 & 45.55 & Kruskal-Wallis $\chi^{2}(2)=10.747$ & .005 & $\begin{array}{l}\text { First-episode AN }>\text { controls: } p=.01, \text { abs }(\mathrm{r})=.3 \\
\text { Recovered }>\text { controls: } p=.002 \text {, abs }(\mathrm{r})=.4 \\
\text { First-episode } \mathrm{AN}<\text { Recovered ns }\end{array}$ \\
\hline $\begin{array}{l}\text { ADOS item: B10, Amount of Social } \\
\text { Overtures, mean rank }\end{array}$ & 58.59 & 69.75 & 45.26 & Kruskal-Wallis $\chi^{2}(2)=12.918$ & .002 & $\begin{array}{l}\text { First-episode AN }>\text { controls: } p=.02 \text {, abs }(\mathrm{r})=.2 \\
\text { Recovered }>\text { controls: } p<.001, \text { abs }(\mathrm{r})=.4 \\
\text { First-episode } \mathrm{AN}<\text { Recovered ns }\end{array}$ \\
\hline $\begin{array}{l}\text { ADOS item: B12, Amount of } \\
\text { Reciprocal Social Communication, } \\
\text { mean rank }\end{array}$ & 65.64 & 59.54 & 44.84 & Kruskal-Wallis $\chi^{2}(2)=14.606$ & .001 & $\begin{array}{l}\text { First-episode AN }>\text { controls: } p>.001 \text {, abs }(\mathrm{r})=.4 \\
\text { Recovered }>\text { controls: } p=.006, \text { abs }(\mathrm{r})=.3 \\
\text { First-episode AN }>\text { Recovered ns }\end{array}$ \\
\hline $\begin{array}{l}\text { ADOS item: B13, Overall Quality of } \\
\text { Rapport, mean rank }\end{array}$ & 60.98 & 63.36 & 47.12 & Kruskal-Wallis $\chi^{2}(2)=8.119$ & .02 & $\begin{array}{l}\text { First-episode AN }>\text { controls: } p=.01 \text {, abs }(\mathrm{r})=.2 \\
\text { Recovered }>\text { controls: } p=.01 \text {, abs }(\mathrm{r})=.3 \\
\text { First-episode } \mathrm{AN}<\text { Recovered ns }\end{array}$ \\
\hline \multicolumn{7}{|l|}{ Vineland-II and social cognition tests ${ }^{\mathrm{e}}$} \\
\hline $\begin{array}{l}\text { Vineland II Socialization Domain, } \\
\text { Index score }\end{array}$ & $106.1(10.5)$ & $108.8(9.1)$ & $111.8(4.9)$ & $\begin{array}{l}\mathrm{F}(2,90): 3.794 \\
\mathrm{R}^{2}=.08\end{array}$ & .03 & $\begin{array}{l}\text { First-episode AN }<\text { controls: } p=.009, d=-.7 \\
\text { Recovered }<\text { controls ns } \\
\text { First-episode AN }<\text { Recovered ns }\end{array}$ \\
\hline MiniPONS Total, correct answers* & $35.6(3.2)$ & $32.5(4.6)$ & $33.9(3.3)$ & $\begin{array}{l}\mathrm{F}(2,109): 6.666 \\
\mathrm{R}^{2}=.11\end{array}$ & .002 & $\begin{array}{l}\text { First-episode AN>controls: } p=.05, d=.5 \\
\text { Recovered }<\text { controls ns } \\
\text { First-episode AN }>\text { Recovered: } p=.008, d=.8\end{array}$ \\
\hline
\end{tabular}




\begin{tabular}{|c|c|c|c|c|c|c|}
\hline Exploratory measures & $\begin{array}{l}\text { First-episode } \\
\text { AN }(N=43)\end{array}$ & $\begin{array}{l}\text { Recovered } \\
\text { AN }(\mathrm{N}=28)\end{array}$ & Controls $(\mathrm{N}=41)$ & Test statistics $^{\mathrm{a}}$ & $p$ & 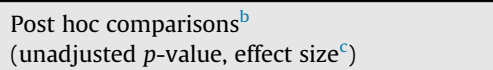 \\
\hline $\begin{array}{l}\text { MiniPONS Body Gestures, } \\
\text { correct answers* }\end{array}$ & $9.2(1.3)$ & $7.7(1.9)$ & $9.0(1.3)$ & $\begin{array}{l}\mathrm{F}(2,109): 9.161 \\
\mathrm{R}^{2}=.14\end{array}$ & $<.001$ & $\begin{array}{l}\text { First-episode AN }>\text { controls ns } \\
\text { Recovered }<\text { controls: } p=.01, d=-.8 \\
\text { First-episode AN }>\text { Recovered: } p=.003, d=.9\end{array}$ \\
\hline $\begin{array}{l}\text { MiniPONS Facial Expression, } \\
\text { correct answers* }\end{array}$ & $5.0(1.1)$ & $4.6(1.4)$ & $5.0(1.1)$ & $\begin{array}{l}F(2,109): 1.431 \\
R^{2}=.03\end{array}$ & .24 & \\
\hline $\begin{array}{l}\text { MiniPONS Vocal Prosody, } \\
\text { correct answers* }\end{array}$ & $11.1(1.4)$ & $10.1(2.0)$ & $10.3(1.7)$ & $\begin{array}{l}\mathrm{F}(2,109): 3.939 \\
\mathrm{R}^{2}=.07\end{array}$ & .02 & $\begin{array}{l}\text { First-episode AN }>\text { controls ns } \\
\text { Recovered }<\text { controls ns } \\
\text { First-episode AN }>\text { Recovered: } p=.04, d=.6\end{array}$ \\
\hline $\begin{array}{l}\text { MiniPONS Facial Expression, and } \\
\text { Vocal Prosody correct answers* }\end{array}$ & $10.3(1.5)$ & $10.1(1.2)$ & $9.6(1.4)$ & $\begin{array}{l}\mathrm{F}(2,109): 2.755 \\
\mathrm{R}^{2}=.05\end{array}$ & .07 & \\
\hline RME-R, correct answers* & $25.6(3.2)$ & $23.8(2.7)$ & $24.1(3.7)$ & $\begin{array}{l}\mathrm{F}(2,109): 3.223 \\
\mathrm{R}^{2}=.06\end{array}$ & .04 & $\begin{array}{l}\text { First-episode AN }>\text { controls ns } \\
\text { Recovered }<\text { controls ns } \\
\text { First-episode AN }>\text { Recovered ns }\end{array}$ \\
\hline AT Film type, correct answers* & $2.6(.7)$ & $2.4(.9)$ & $2.7(.7)$ & Kruskal-Wallis $\chi^{2}(2)=7.997$ & .24 & \\
\hline $\begin{array}{l}\text { AT feelings questions, correct } \\
\text { answers }\end{array}$ & $6.4(1.3)$ & $6.3(1.2)$ & $6.1(1.2)$ & $\begin{array}{l}\mathrm{F}(2,109): .513 \\
\mathrm{R}^{2}=.01\end{array}$ & .60 & \\
\hline TASIT Total, correct answers* & $33.9(3.2)$ & $32.4(2.8)$ & $33.4(3.7)$ & $\begin{array}{l}\mathrm{F}(2,109): 1.935 \\
\mathrm{R}^{2}=.03\end{array}$ & .15 & \\
\hline TASIT Sincere, correct answers* & $16.4(2.3)$ & $15.8(2.9)$ & $16.2(3.2)$ & Kruskal-Wallis $\chi^{2}(2)=.726$ & .70 & \\
\hline TASIT Sarcastic, correct answers* & $17.5(1.9)$ & $16.6(2.1)$ & $17.2(2.1)$ & $\begin{array}{l}F(2,109): 1.795 \\
R^{2}=.03\end{array}$ & .17 & \\
\hline AGN, difference in correct latency & $9.2(31.7)$ & $5.7(31.1)$ & $10.2(34.8)$ & $\begin{array}{l}\mathrm{F}(2,103): .161 \\
\mathrm{R}^{2}=.003\end{array}$ & .85 & \\
\hline
\end{tabular}

$*$ = reduced test sets excluding items with ceiling effect. ADOS = Autism Diagnostic Observation Schedule; AGN = CANTAB Affective Go/No-go test; AT = Animated Triangles; BAI-Y = Beck Anxiety Inventory Youth; $\mathrm{BDI}-\mathrm{Y}=$ Beck Depression Inventory Youth; BMI = body mass index; $\mathrm{EDE}=$ Eating Disorder Examination; MiniPONS = Profile of Nonverbal Sensitivity, short version; $\mathrm{n} / \mathrm{a}=$ not applicable; $\mathrm{ns}=$ non-significant; $\mathrm{RME}-\mathrm{R}=$ Reading-the-Mind-in-the-Eyes Revised; SD = standard deviation; TASIT = The Awareness of Social Inference Test.

a One-way ANOVA if not otherwise specified.

b Post hoc analyses for parametric tests: Tukey or Games-Howell, for nonparametric tests: Mann-Whitney U Test.

c Poffect size: $\mathrm{d}=$ Cohen's d. $\mathrm{r}$ calculated as $\mathrm{r}=$ standardized test statistic//VN, reported in absolute values.

d Only displaying those ADOS items (communication and social interaction clusters), where one or both clinical groups displayed a distribution significantly different from controls (after post hoc Bonferroni correction).

MiniPONS Total and TASIT total are not used in analyses, but are included here for information 


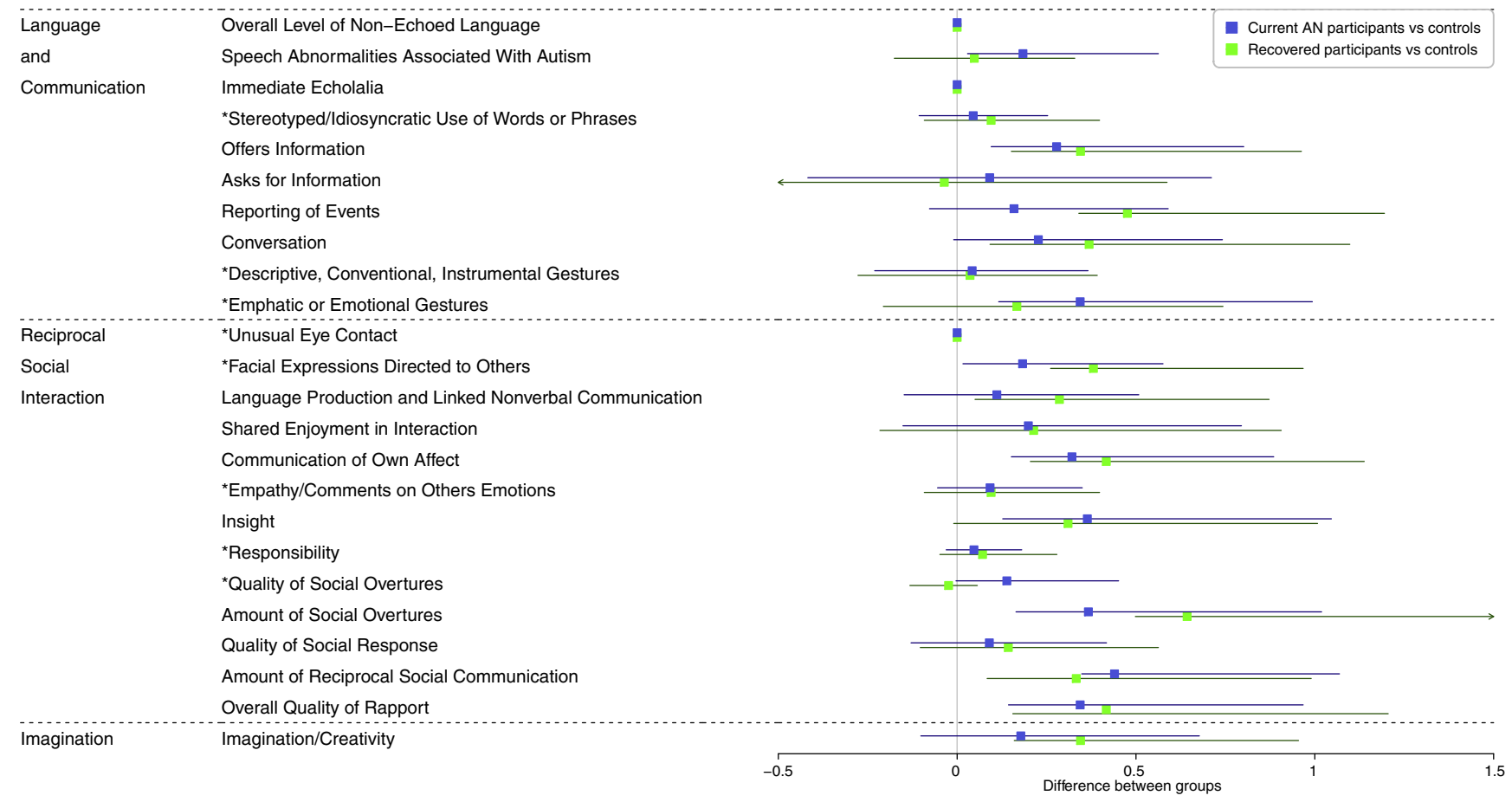

Figure 2. Comparing first-episode AN and recovered participants with controls; mean difference and confidence intervals for ADOS single items. Vertical axis (marked 0 ) represents no difference to controls, right-hand side of the vertical axis represents higher mean scores (more impairment) than controls, left-hand side of the vertical axis represents lower mean scores (less impairment) than controls. Family-wise Bonferroni corrected confidence intervals shown: for "Language and Communication" cluster 99.5\%, for "Reciprocal Social Interaction" cluster 99.6\%, for "Imagination" 95\%. Items from clusters "Restricted Interests," "Stereotyped Behaviors," and "Other Abnormal Behaviors" are not shown due to lack of variation. "Items included in the ADOS-2 algorithms.

Reduced social function has not been previously documented in individuals fully recovered from AN. Other studies suggest that difficulties in social and adaptive function may persist after recovery from adolescent AN [29]. In this regard, our finding extends earlier reports and suggests that impairments in social function may persist, also after recovery.

Possibly, social difficulties in AN may in fact reflect other factors, such as social anxiety and emotional avoidance, possibly reinforced by starvation, but be mislabeled as autistic traits [30]. In the present study, however, social function was not associated with BMI percentile, eating disorder symptoms, depression, or anxiety symptoms. Alexithymia, which is well documented in individuals with AN, may also affect social function [31]. Indeed, groups differed on the ADOS item Communication of Own Affect (an item not included in the ADOS algorithm). However, the interactional style in both AN groups indicated impairment of several other social behaviors, such as Amount of Social Overtures and Amount of Reciprocal Social Communication, suggesting that alexithymia may not explain the entire impairment measured during social interaction.

Participants with first-episode AN performed equal to or better than controls in tests of social cognition. This somewhat perplexing observation is not in line with studies of social cognition in adults with current AN [4,5]. Indeed, a meta-analysis of the RME-R confirmed reduced performance in adults with AN [4]. Duration of illness might explain the difference in RME-R performance [32]. The average duration of illness in our firstepisode AN participants was shorter $(<1$ year) than that reported in prior studies of adults (duration between 3.6 and 9.9 years) [4,5]. Interestingly, a functional magnetic resonance imaging (fMRI) study in adolescents with current AN reported normal verbal performance in a ToM task but reduced activation of brain networks supporting ToM [33]. Moreover, reduced fMRI activation during the acute phase in that study was associated with poorer outcome a year later. This finding may suggest that differences in social information processing, which are too subtle to measure with a behavioral task, may already be present in the early phases of AN and that individuals with such impairments are at risk for a longer duration of illness and therefore might be over-represented in samples of adults with AN.

The recovered participants displayed a few deficits in social cognition; they performed lower than both other groups on the MiniPONS body gesture, and lower than first-episode AN participants on vocal prosody. The MiniPONS test has not been used in individuals with AN before, but another study of social perception in AN used the Interpersonal Perception Task and described a trend toward reduced performance in adult participants with current AN [34]. The difference in social perception between our recovered and first-episode AN participants corroborates the hypothesis that social cognitive deficits might be associated with duration of illness. Alternatively, the younger mean age at AN onset in our recovered participants might have struck a critical "window" of development where the illness impeded their learning of social perception skills.

\section{Strengths and limitations}

A strength of our study is that the first-episode AN participants were recently diagnosed young females, consecutively 
recruited, and the recovered participants met strict criteria for recovery. Unfortunately, consideration of participants' treatment sessions, education, and unplanned cancellations induced a considerable variation among participants with regard to the time span between first and last testing session. Although groups did not differ in this aspect, we cannot rule out that the medical condition of participants with first-episode AN might have changed between sessions. It would be preferable to have equal sample sizes, age matching, and a contrast group with ASD without AN, along with blinding of raters, but this was not possible. More sensitive tests of social cognition might detect more subtle differences between groups. Owing to the low number of participants with social anxiety disorder in the sample, it is not likely that our findings were influenced by social anxiety, and symptoms of anxiety did not explain the ADOS score. However, it would be most valuable for future studies to characterize the group of individuals with AN and co-occurring anxiety disorders further. Moreover, larger samples would allow comparison between individuals with different subtypes of AN or different profiles of symptoms to identify a particular subgroup in need of treatment for their social function impairment. Finally, with the cross-sectional design, we cannot be entirely sure that the two groups of affected participants did not differ with respect to severity of AN at onset, and this study cannot determine the state or trait nature of the observed deficits in social function.

We thus conclude that both individuals with first-episode AN and those recovered from AN on a group level displayed impairment of social function, even in the absence of a diagnosis of autism or Asperger syndrome. These impairments of social function were not explained by symptoms of anxiety or depression. In participants with first-episode AN, impairments of social function were not related to symptom severity of AN. However, we cannot discern whether our findings of normal performance of social cognition tasks may reflect a lack of sensitivity of the tests in this specific group of participants or whether the observed impairment of social function may be related to other factors than those tapped by tests of social cognitive function (e.g., factors of "nonsocial" cognition).

The presence of social function deficits in other patient populations has led to targeted interventions to enhance social skills [35]. In young individuals with AN, it is crucial for prognosis to address the core symptoms and secure weight restoration [36], for example, in the context of family-based treatment (FBT), which is the recommended first line of treatment for the young [37]. Impairments in social function, such as understanding and interpreting social cues, however, may hamper treatment response in FBT by evoking misunderstandings and feelings of disempowerment. FBT for youth with AN might be enhanced by integrating explicit psychoeducation and behavioral strategies to enhance intrafamilial interaction. In the later phases, interventions that specifically address social skills may further facilitate recovery. The significance of the communication with peers, both gestures of acceptance and rejection, becomes more central during the teenage years. Focusing on enhancing social function in this age group may thus facilitate the social reintegration of the patients, such that social relations may take over the fundamental importance that ruminations of thoughts about eating and body shape have provided during disorder.

Several treatment strategies aimed at adults target social skills and may well be adapted to the subgroup of young patients with deficits in social function, such as Interpersonal Therapy
[38], the "Maudsley Model of Anorexia Nervosa Treatment for Adults" [39], or the "Radically Open-Dialectic Behavior Therapy" [40]. Future research should target the potential impact of social function as a moderator for treatment efficacy and as a predictor for relapse in young people with AN because this area still lacks evidence from rigorous empirical studies.

\section{Acknowledgments}

The authors thank Christine Lund, M.S. Psych., for backtranslation of target words and phrases in MiniPONS, RME-R, and AT questions; M.S. student of physics Laurids Møller Jepsen for programming the RME-R for electronic administration; M.S. student of psychology Nanna Romar Pagsberg for pilot testing MiniPONS and AT questions; and Klaus Kaae-Andersen, M.S. Stats., Ph.D., for statistical consultation. Furthermore, they thank Stolpegaard Psychotherapy Centre, Mental Health Services-the Capital Region of Denmark, for extending our invitation to adults with first-episode AN. Finally, they thank all participants and parents, who took the time and effort to participate in this study.

\section{Funding Sources}

G.J.'s Fond, Danielsens Fond, Fru C. Hermansens Mindelegat, Beatrice Surovell Haskell Fund for Child Mental Health Research in Copenhagen, and Mental Health Services in the Capital Region of Denmark supported this work. Dr. C.M.B. acknowledges funding from the Swedish Research Council (VR Dnr: 5382013-8864).

\section{References}

[1] Treasure J, Schmidt U. The cognitive-interpersonal maintenance model of anorexia nervosa revisited: A summary of the evidence for cognitive, socioemotional and interpersonal predisposing and perpetuating factors. J Eat Disord 2013;1:13.

[2] Adolphs R. Cognitive neuroscience of human social behaviour. Nat Rev Neurosci 2003;4:165-78.

[3] Brothers L. The social brain: A project for integrating primate behavior and neurophysiology in a new domain. In: Cacioppo JT, Berntson GG, Adolphs R, et al., eds. Foundations in Social Neuroscience. 1st edition. 1990:27-51.

[4] Oldershaw A, Hambrook D, Stahl D, et al. The socio-emotional processing stream in anorexia nervosa. Neurosci Biobehav Rev 2011;35:970-88.

[5] Caglar-Nazali HP, Corfield F, Cardi V, et al. A systematic review and metaanalysis of "Systems for Social Processes" in eating disorders. Neurosci Biobehav Rev 2014;42:55-92.

[6] Lord C, Rutter M, DiLavore PC, et al. Autism diagnostic observation schedule, 2nd edition. (ADOS-2) Vejledning (Del 1): Modul 1-4 København: Hogrefe Psykologisk Forlag; 2013.

[7] Fairburn CG. Cognitive behavior therapy and eating disorders. New York: Guilford Press; 2008.

[8] Morgan HG, Hayward AE. Clinical assessment of anorexia nervosa. The Morgan-Russell outcome assessment schedule. Br J Psychiatry J Ment Sci 1988;152:367-71.

[9] Herpertz-Dahlmann B, Müller B, Herpertz S, et al. Prospective 10-year follow-up in adolescent anorexia nervosa-course, outcome, psychiatric comorbidity, and psychosocial adaptation. J Child Psychol Psychiatry 2001; 42:603-12.

[10] Rutter M, Bailey A, Lord C. Social communication questionnaire (SCQ). Los Angeles: West. Psychol. Serv; 2003. n.d.

[11] Kopp S, Gillberg C. The Autism Spectrum Screening Questionnaire (ASSQ)Revised Extended Version (ASSQ-REV): An instrument for better capturing the autism phenotype in girls? A preliminary study involving 191 clinical cases and community controls. Res Dev Disabil 2011;32:2875-88.

[12] Lord C, Rutter M, Le Couteur A. Autism diagnostic interview-revised: A revised version of a diagnostic interview for caregivers of individuals with possible pervasive developmental disorders. J Autism Dev Disord 1994;24:659-85.

[13] Kaufman J, Birmaher B, Brent DA, et al. K-SADS-PL. J Am Acad Child Adolesc Psychiatry 2000;39:1208. 
[14] Beck JS, Beck AT, Jolly JB, Steer RA. Beck youth inventories second edition. Minneapolis: Pearson Inc; 2005.

[15] Reynolds CR, Kamphaus RW. Reynolds Intellectual Assessment Scales ${ }^{\mathrm{TM}}$ (RIAS $^{\text {TM }}$ ). Lutz, FL: Psychological Assessment Resources, Inc; 2003.

[16] Lord C, Risi S, Lambrecht L, et al. The autism diagnostic observation schedule-generic: A standard measure of social and communication deficits associated with the spectrum of autism. J Autism Dev Disord 2000; 30:205-23.

[17] Sparrow SS, Cicchetti DV, Balla DA. Vineland-II Vineland adaptive behavior scales, second edition. Minneapolis: Pearson Inc; 2011.

[18] Green MF, Olivier B, Crawley JN, et al. Social cognition in Schizophrenia: Recommendations from the measurement and treatment research to improve cognition in schizophrenia new approaches conference. Schizophr Bull 2005;31:882-7.

[19] Baron-Cohen S, Wheelwright S, Hill J, et al. The "reading the mind in the eyes" test revised version: A study with normal adults, and adults with Asperger syndrome or high-functioning autism. J Child Psychol Psychiatry 2001;42:241-51.

[20] Bänziger T, Scherer KR, Hall JA, Rosenthal R. Introducing the MiniPONS: A short multichannel version of the Profile of Nonverbal Sensitivity (PONS). J Nonverbal Behav 2011;35:189-204.

[21] White SJ, Coniston D, Rogers R, Frith U. Developing the Frith-Happe animations: A quick and objective test of theory of mind for adults with autism. Autism Res Off J Int Soc Autism Res 2011;4:149-54.

[22] Bliksted V, Fagerlund B, Weed E, et al. Social cognition and neurocognitive deficits in first-episode schizophrenia. Schizophr Res 2014;153:9-17.

[23] P. Fray, T.W. Robbins, B.J. Sahakian, Neuropsychiatric applications of CANTAB. 1996.

[24] Hus V, Lord C. The autism diagnostic observation schedule, module 4: Revised algorithm and standardized severity scores. J Autism Dev Disord 2014;44:1996-2012.

[25] Huke V, Turk J, Saeidi S, et al. Autism spectrum disorders in eating disorder populations: A systematic review. Eur Eat Disord Rev 2013;21: 345-51.

[26] Mandy W, Tchanturia K. Do women with eating disorders who have social and flexibility difficulties really have autism? A case series. Mol Autism 2015;6:6.

[27] Pooni J, Ninteman A, Bryant-Waugh R, et al. Investigating autism spectrum disorder and autistic traits in early onset eating disorder. Int J Eat Disord 2012;45:583-91.
[28] Rhind C, Bonfioli E, Hibbs R, et al. An examination of autism spectrum traits in adolescents with anorexia nervosa and their parents. Mol Autism 2014; 5:56.

[29] Halvorsen I, Andersen A, Heyerdahl S. Girls with anorexia nervosa as young adults. Eur Child Adolesc Psychiatry 2005;14:397-406.

[30] Zucker NL, Losh M, Bulik CM, et al. Anorexia nervosa and autism spectrum disorders: Guided investigation of social cognitive endophenotypes. Psychol Bull 2007:133:976-1006.

[31] Nowakowski ME, McFarlane T, Cassin S. Alexithymia and eating disorders: A critical review of the literature. J Eat Disord 2013;1:21.

[32] Harrison A, Sullivan S, Tchanturia K, Treasure J. Emotion recognition and regulation in anorexia nervosa. Clin Psychol Psychother 2009;16: $348-56$.

[33] Schulte-Rüther M, Mainz V, Fink GR, et al. Theory of mind and the brain in anorexia nervosa: Relation to treatment outcome. J Am Acad Child Adolesc Psychiatry 2012;51:832-841.e11.

[34] Renwick B, Dejong H, Kenyon M, et al. Social perception in people with eating disorders. Eur Psychiatry J Assoc Eur Psychiatr 2013;28:436-41.

[35] Horan WP, Kern RS, Tripp C, et al. Efficacy and specificity of social cognitive skills training for outpatients with psychotic disorders. J Psychiatr Res 2011;45:1113.

[36] Madden S, Miskovic-Wheatley J, Wallis A. Early weight gain in family-based treatment predicts greater weight gain and remission at the end of treatment and remission at 12-month follow-up in for adolescent anorexia nervosa. Int J Eat Disord 2015;48:919-22. Wiley Online Library (n.d.).

[37] Couturier J, Kimber M, Szatmari P. Efficacy of family-based treatment for adolescents with eating disorders: A systematic review and meta-analysis. Int J Eat Disord 2013;46:3-11.

[38] Rieger E, Van Buren DJ, Bishop M, et al. An eating disorder-specific model of interpersonal psychotherapy (IPT-ED): Causal pathways and treatment implications. Clin Psychol Rev 2010;30:400-10.

[39] Schmidt U, Magill N, Renwick B, et al. The Maudsley Outpatient Study of Treatments for Anorexia Nervosa and Related Conditions (MOSAIC): Comparison of the Maudsley Model of Anorexia Nervosa Treatment for Adults (MANTRA) with Specialist Supportive Clinical Management (SSCM) in outpatients with broadly defined anorexia nervosa: A randomized controlled trial. J Consult Clin Psychol 2015;83:796-807.

[40] Lynch TR, Gray KL, Hempel RJ, et al. Radically open-dialectical behavior therapy for adult anorexia nervosa: Feasibility and outcomes from an inpatient program. BMC Psychiatry 2013;13:293. 